\title{
The impact of the COVID-19 lockdown on human psychology and physical activity; a space analogue research perspective
}

\author{
Jeroen Van Cutsem ${ }^{1,2}$ (D), Vera Abeln ${ }^{3}$ (D), Stefan Schneider ${ }^{3,4}$, Nathan Keller ${ }^{5}$, Ana Diaz-Artiles ${ }^{5,6}$, \\ Miguel A. Ramallo ${ }^{7}$, Emilie Dessy ${ }^{1}$, Nathalie Pattyn ${ }^{1,2}$, Fabio Ferlazzo $^{8^{*}}$ and Gabriel G. De La Torre ${ }^{7 *}$ \\ ${ }^{1}$ VIPER Research Unit, Royal Military Academy, Brussels, Belgium \\ ${ }^{2}$ Human Physiology and Sports Physiotherapy Research Group, Vrije Universiteit Brussel, Brussels, Belgium \\ ${ }^{3}$ Institute of Movement and Neurosciences, German Sport University, Cologne, Germany \\ ${ }^{4}$ VasoActive Research Group, School of Health and Sport Sciences, University of the Sunshine Coast, Queensland, Australia \\ ${ }^{5}$ Department of Health and Kinesiology, Texas A\&M University, College Station, TX, USA \\ ${ }^{6}$ Department of Aerospace Engineering, Texas A\&M University, College Station, TX, USA \\ ${ }^{7}$ Neuropsychology and Experimental Psychology Lab, University of Cadiz, 11510 Puerto Real, Spain \\ ${ }^{8}$ Department of Psychology, Sapienza University of Rome, Rome, Italy \\ Author for correspondence: Jeroen Van Cutsem, E-mail: Jvcutsem91@gmail.com
}

Received: 21 June 2021; Revised: 19 October 2021; Accepted: 17 December 2021

Keywords: COVID-19, Isolated and confined environment, lockdown, physical activity, sleep, space analogue

\begin{abstract}
Introduction. Astronauts will encounter isolated, confined and extreme (ICE) conditions during future missions, and will have to be able to adapt. Until recently, however, few places on Earth could serve as acceptable space analogues (i.e., submarine and polar regions). The coronavirus disease-2019 (COVID-19)-related lockdowns around the globe provided a good opportunity to obtain more comprehensive datasets on the impact of prolonged isolation on human functioning in a very large sample.

Methods. Seven hundred forty-eight individuals (Belgium 442, Spain 183, Germany 50, Italy 50, US 23; Mean age \pm SD: $41 \pm 14$ years, with an age range of $18-83$ years; $66 \%$ women) filled out an online survey assessing the impact of the COVID-lockdown on psychological, exercise and general health variables a first time near the beginning of the initial lockdown (hereafter 'T1'; $24 \pm 13$ days after the start of the first lockdown; i.e., 3 weeks after the start of the first lockdown) and a second time a couple of weeks thereafter (hereafter 'T2'; $17 \pm 5$ days after the first online survey; i.e., 6 weeks after the start of the first lockdown).

Results. From T1 to T2 an improvement of subjective sleep quality was observed $(P=0.003)$, that was related to an increase in subjective sleep efficiency and a decrease in sleep latency and disturbance $(P \leq 0.013)$. Weekly sitting time decreased, and the weekly amount of moderate and vigorous physical activity increased from T1 to T2 $(P \leq 0.049)$. No differences from T1 to T2 were observed in terms of mood, loneliness and state anxiety. A lower amount of sitting time was significantly correlated with improved subjective sleep quality $(r=0.096$, $P=0.035)$ and with an increased amount of moderate $(r=-0.126, P=0.005)$ and vigorous $(r=-0.110$, $P=0.015)$ physical activity.

Conclusion. Compared to 3 weeks into the first COVID-imposed lockdown, 6-weeks after the start of the first COVID-imposed lockdown, physical activity and subjective sleep scores were positively impacted. The present, large sample size study further confirms exercise as a worthwhile countermeasure to psycho-physiological deconditioning during confinement.
\end{abstract}

*Fabio Ferlazzo and Gabriel G. De La Torre share the last author-position. 


\section{Contents}

Introduction

Methods

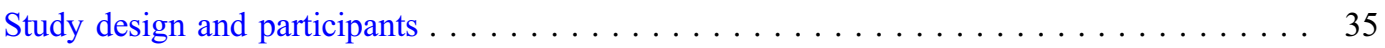

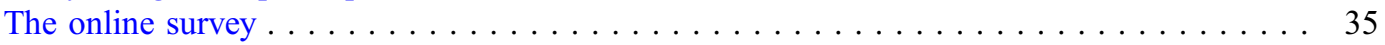

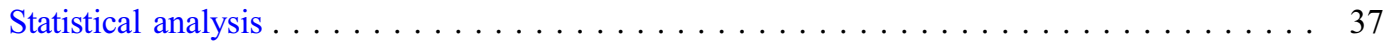

$\begin{array}{ll}\text { Results } & \mathbf{3 7}\end{array}$

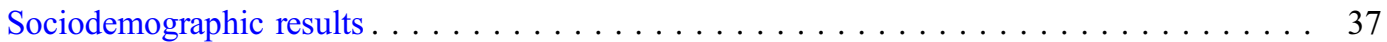

The effect of prolonged COVID restrictions on human functioning and psychophysiology . . 38

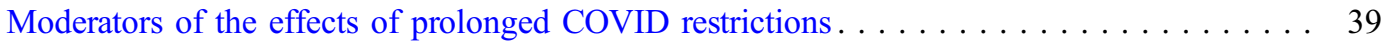

Discussion $\quad 41$

The effect of prolonged COVID restrictions on human functioning and psychophysiology . . 41

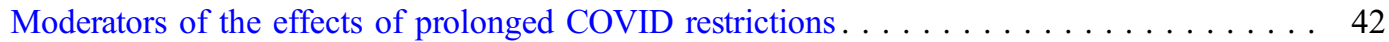

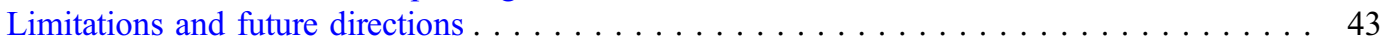

$\begin{array}{ll}\text { Conclusion } & 44\end{array}$

\section{Introduction}

Astronauts, both during short and long-duration space exploration missions, will encounter isolated, confined and extreme (ICE) conditions, and will have to be able to adapt as effectively as possible to get the most out of their mission and accomplish mission objectives (Bartone et al., 2018). ICE environments are very complex environments in which various types of stressors constantly and simultaneously affect human functioning (i.e., physical and mental performance) and psychophysiology. Knowledge about the relative weight of each factor that makes up an ICE environment, as well as about the impact of an ICE environment on human functioning and psychophysiology is of paramount importance to develop effective countermeasures and to ensure mission success (Zivi et al., 2020). For example, factors that are known to play a role in the effect of an ICE environment on human functioning during space missions include long-term microgravity, constant noise, radiation and prolonged isolation (Zivi et al., 2020). Currently, it is well known that interindividual differences exist in how quickly one adapts to ICE conditions. The underlying mechanisms of these interindividual differences are however less clear (Bartone et al., 2018). Enhancing our understanding of how these stressors affect human functioning is necessary to meet the critical need to predict the time course, magnitude and individual variability in behavioural, cognitive, affective and interpersonal reactions of astronauts for future long-term mission planning.

Prolonged isolation is possibly one of the most stressing factors that make up an ICE environment (Kanas, 1998, Pagel and Choukèr, 2016). During space missions, prolonged isolation not only entails the deprivation of social activities, touch and speech, but also a heavy burden of responsibility to make crucial decisions without the benefit of feedback or a second opinion (Urbina and Charles, 2014). Within NASA's human research programme, isolation has been identified as one of the five hazards of human spaceflight (https://www.nasa.gov/hrp/5-hazards-of-human-spaceflight). Multiple space analogue mission studies have already been conducted specifically focusing on the role of prolonged isolation and confinement on operational performance and on interpersonal issues that may be encountered during small-group (space) missions (Basner et al., 2014; Pagel and Choukèr, 2016). Mairesse et al. (2019) for example followed 13 males during their 13-month winter-over campaign in Antarctica and reported that human sleep and performance were impaired. The negative effect of prolonged isolation on human functioning is further confirmed by Sandal et al. (2018), who suggested that individuals enter a state of psychological hibernation as a stress-coping mechanism during an Antarctic over-wintering. Moreover, Abeln et al. (2015) could show that individuals with regular exercise routine maintained their psychological state during Antarctic overwintering as opposed to their sedentary crewmembers, who showed serious deteriorations. In terms of sleep in Antarctica, the review of Pattyn et al. 
(2018) could however not confirm a relation between decreased physical activity and the occurrence of sleep disturbances. Nevertheless, also in the review of Pattyn et al. (2018) exercise was put forward as an effective countermeasure for sleep disorders. In addition, Matsangas et al. (2017) identified, over 1 month, substantial individual differences in sleep-related behaviour, physical activity and exposure to light between crewmembers of the Hawaii Space Exploration Analog and Simulation (HI-SEAS). Recently, Mimoso et al. (2020) also reported that, during 4 months of a ground-based analogue space mission (i.e., SIRIUS-19 analogue campaign), crew performance during a teleoperation task was linked with confinement stressors.

There are only a few places on Earth to simulate realistic isolations conditions and therefore, are considered good ICE space analogues (i.e., submarine, deserted, volcanic and especially polar (Arctic and Antarctic) regions). This fact highlights one of the main difficulties in human performance research in the space and space analogue domain: the small sample size. Crews usually consist of less than six people, owing on the one hand to the remoteness of the areas where space analogue studies are carried out, and on the other hand due to the specialization and high training profile of astronauts participating in crewed space missions. Working with small sample sizes eventually results in a lack of big, normative, datasets from larger samples in both clinical and healthy populations.

Recently, however, the coronavirus disease-2019 (COVID-19) pandemic has arisen and affected our world and way of living substantially. At the beginning of the COVID-19 pandemic in Europe, around March 2020, several countries adopted different degrees of home and business lockdown and confinement measures. Suddenly millions of people across Europe and later in the United States and other countries were forced into a confinement situation similar to China's earlier efforts. The World Health Organization recommended various protective measures to stop the transmission of COVID-19 including restriction of social interaction, face masks and other health-related rules. Governments of many EU countries opted for confinement, allowing citizens to go out of their homes only for very specific reasons and circumstances. As such, the COVID-19 pandemic turned the world into a real-life prolonged isolated environment. This provided, besides all the negative effects on the world, a unique opportunity to obtain normative data related to the effect of prolonged isolation on psychological, exercise and general health variables from a very large sample of people living across Europe and the rest of the world.

One of the consequences of COVID-19 lockdowns, which is also applicable to space analogues, relates to physical activity constraints. During space missions, tight constraints upon the use of exercise exist due to different causes: working space availability, environmental aspects $\left(\mathrm{CO}_{2}\right.$, heat, etc.), logistical and operational aspects (Scott et al., 2019; Keller et al., 2021). Some of these tight constraints were (unwillingly) replicated in COVID-19 lockdown periods. People encountered problems continuing their regular physical exercise routines as gyms, pools and many other sport venues were closed and time outdoors was limited. Apart from the fact that there were similar physical activity limitations during the COVID-19 lockdown and during a space mission, other similarities could be identified as well. Factors that affect sleep in space include photoperiodic alterations, microgravity, environmental discomfort (e.g., confinement), psychological stressors (e.g., stress, anxiety), work demands (slam shifts) and decreased physical activity (Mairesse et al., 2019). Like for physical activity, some of these factors were (unwillingly) replicated/triggered in COVID-19 lockdown periods (e.g., environmental discomfort, psychological stress and decreased physical activity).

Therefore, to create further insight into the factors playing a role in the presence of interindividual differences in the effect that prolonged isolation has on human functioning and psychophysiology, the present study aimed to investigate the effects of the COVID-related lockdown on psychological, exercise and general health variables from a very large sample of people living across Europe and the U.S., during the first 6 weeks of the very first lockdown. Based on the above-mentioned studies, we hypothesized that, on average, a prolonged COVID-related lockdown (i.e., 6 weeks of lockdown vs 3 weeks of lockdown) would exert a negative effect on human psychological functioning and on exercise-related and general health-related variables. In particular, we further hypothesized that sleep quality 
(Gemignani et al., 2014; Matsangas et al., 2017) as well as physical activity (Belavý et al., 2013) would decrease with prolonged COVID-related lockdown.

\section{Methods}

\section{Study design and participants}

Data in the present study were collected by an international team, cooperating to assess the impact of the COVID-19 lockdown measures on psychological, exercise and general health variables. The study was approved by multiple ethical committees (Spain, CEI Cadiz: Psicosoc-covid19-0774N20; USA, Texas A\&M University: IRB2020-0506 M; Italy, Sapienza University: IRB prot. 0000586). To evaluate the effect of prolonged isolation on human functioning and psychophysiology, an online survey, in the native language of each country, was developed and subsequently distributed through social media and university platforms across five different countries: Belgium, Germany, Spain, Italy and USA. This survey was distributed a first time shortly after the beginning of the first lockdown in each specific country (hereafter 'T1'; $24 \pm 13$ days after the start of the first lockdown; i.e., 3 weeks after the start of the first lockdown) and a second time a couple of weeks thereafter (hereafter 'T2'; $17 \pm 5$ days after the first online survey; i.e., 6 weeks after the start of the first lockdown), to all individuals who filled out the first online survey. Ultimately, 748 individuals that completed both the first and second online survey were included in the present study (Belgium 442, Spain 183, Germany 50, Italy 50, US 23). The mean age of this selected sample was $41 \pm 14$ years old, with an age range of $18-83$ years. Men made up $34 \%$ of the sample and had a mean age of $44 \pm 14$ years, women had a mean age of $40 \pm 14$ years.

The start of the first COVID-related lockdown, i.e., the most severe COVID-related restrictions, occurred approximately at the same time in all participating countries: Belgium 18 March 2020, Germany 13 March 2020, Italy 8 March 2020, Spain 13 March 2020 and USA 16 March 2020. COVID-related restrictions slightly varied amongst countries but in general encompassed the following closures: business (i.e., mandatory/recommended home office), schools, sports (e.g., gyms, pools and team sport clubs) and other services (i.e., transportation), social contact-restrictions and travel restrictions. In addition, it was mandatory to wear a mouth mask in all countries, what may be seen as an extra stress factor. Most restrictions remained in place until the end of May 2020 and thus, throughout the follow-up period (T2) of our longitudinal design.

\section{The online survey}

The interdisciplinary online survey, which was implemented using the Google Forms tool, consisted of the following sections: (1) a sociodemographic section, (2) the Pittsburg Sleep Quality Index (PSQI), (3) the short version of the International Physical Activity Questionnaire (IPAQ), (4) the Profile of Mood States (POMS), (5) the UCLA Loneliness Scale, (6) the State-Trait Anxiety Inventory (STAI), (7) a section on the usage of stimulating substances, (8) a salutogenic section and (9) a COVID-related section. Multiple outcome measures that are included in these sections have been frequently used in space analogue studies (Belavý et al., 2013; Pattyn et al., 2017; Mairesse et al., 2019).

\section{Sociodemographics}

Within the demographical section, participants were asked about their age, gender, country of living, educational level, living situation, household and employment status.

\section{Pittsburgh sleep quality inventory}

The PSQI (Buysse et al., 1989) includes 19 self-assessment questions that combine to give seven components of the overall score, with each component receiving a score of $0-3$. In all cases, a score of 0 indicates no difficulty, while a score of 3 indicates severe difficulty. An overall (i.e., the 19 questions) 
sum of 5 or more indicates a 'bad' sleeper. The original version was designed to measure sleep reports over a 1-month interval. However, in the present study, the PSQI was adapted to refer to a 1-week interval (Beck et al., 2004). Included outcome parameters of the PSQI in the present study were the subscores on all seven PSQI-components (subjective sleep quality, sleep latency (i.e., the time it takes to fall asleep), sleep duration, sleep efficiency (i.e., time in bed divided by sleep duration), sleep disturbance, use of sleep medication and daytime dysfunction), the overall PSQI-score, hours in bed, sleep latency, sleep per night and sleep efficiency.

\section{International physical activity questionnaire: short}

The short version of the IPAQ (Craig et al., 2003) was used to measure the physical activity levels of the participants. This questionnaire consists of seven generic items regarding the last 7 days, assessing the types and intensity of physical activity that the participants performed (i.e., vigorous physical activity, moderate physical activity and walking) and the sitting time spent as part of their daily lives. Outcome parameters of the short-IPAQ were the amount of vigorous-intensity, moderate-intensity, walking, sitting and overall (excluding sitting) activity (in minutes) that was performed over the last 7 days.

\section{Profile of mood states}

The POMS questionnaire was originally developed by McNair et al. (1971). In the current study, the 32-item version of the POMS questionnaire was employed. This POMS version consists of five subscales: tension, depression, anger, fatigue and vigour. All items had to be scored from 0 (not at all) to 4 (extremely). The higher the score on a category, the more participants felt this mood state was present. This questionnaire has been translated and validated in multiple languages (French (Fillion and Gagnon, 1999), Dutch (Wald and Mellenbergh, 1990), English (Terry et al., 2003)).

\section{UCLA loneliness scale}

The UCLA Loneliness scale (Russell et al., 1978) is a 20-item scale designed to measure one's subjective feelings of loneliness as well as feelings of social isolation. Participants rate each item on a 4-point Likert-type scale (0, 'I never feel this way'; 4, 'I often feel this way'). Eventually, all items are summed up to provide an overall loneliness score ranging from 0 to 80 .

\section{State-trait anxiety inventory}

The STAI questionnaire, which was originally developed by Spielberger (1983), is a 40-item self-report measure of anxiety that uses a 4-point Likert-type scale (from 1 to 4 points) for each item. It has two scales: state anxiety, i.e., how one feels at the moment; and trait anxiety, i.e., how one generally feels. Both scales consist of 20 items. In the present study, only the state anxiety section was utilized. The overall score ranges from 20 to 80 , where the higher score indicates greater anxiety. A cutoff point of 39-40 has been suggested to detect clinically significant symptoms for the state anxiety scale, however, other studies have suggested a higher cutoff score of 54-55 for older adults (Julian, 2011).

\section{Stimulating substances}

To assess the usage of stimulating substances, participants were asked about their smoking and drinking (e.g., alcohol and caffeine-containing drinks) habits during the 7 days prior filling out the online survey. In addition, participants were also asked whether they believed the COVID-related lockdown had affected these habits. The outcome parameters of these questions were the average daily intake of cigarettes, cups of coffee, glasses of beer and liquor, glasses of wine and caffeinated energy drinks.

\section{Salutogenic section}

In order to evaluate a potential positive effect of the COVID-related lockdown, participants were asked, with a yes or no question, whether they perceived any positive effect of the present situation on their life in the last week (e.g., more time to exercise, time for family, possibility to participate in online courses). 


\section{COVID-related section}

COVID-related questions were assessed with a 5-point Likert-type scale. Examples include: whether participants 'thought the COVID-crisis would be one of long duration?', whether they 'trust the authorities to handle the crisis as optimally as possible?', and whether they 'perceived COVID-19 to be a serious and life-threatening disease?'. In addition, participants were also asked whether they had contracted COVID-19 themselves, lived with someone who had, or had lost someone close to them to COVID-19.

\section{Statistical analysis}

All data are presented as mean $\pm \mathrm{SD}$, unless stated otherwise. The Kolmogorov Smirnov test, and visual interpretation of histograms as a double check, were used to test the normality of the data. If data were not normally distributed (see Table 1), nonparametric Wilcoxon tests were used to observe the effect of prolonged COVID restrictions (i.e., differences between $\mathrm{T} 1$ and $\mathrm{T} 2$ time points). If data were normally distributed (see Table 2), parametric paired-samples $t$-tests were performed to elucidate the effect of prolonged COVID restrictions. In specific variables (i.e., PSQI total score, vigorous activity score, moderate activity score and sitting time) where an effect of prolonged COVID restrictions was present, a possible role for moderating variables was assessed as follows: for quantitative moderating variables, a Pearson correlation coefficient; for qualitative moderating variables, a $\chi^{2}$ distribution. Included quantitative moderating variables were: age, T1 overall PSQI-score, T1 overall IPAQ-score and T1 STAI-score. Included qualitative moderating variables were: country, categorized age $(<25$ years $=1$; $25-44=2 ; 45-64=3 ;>64=4$ ), gender, categorized educational level (primary education $=0$; secondary education $=1$; bachelor degree $=2$; master degree $=3 ; \mathrm{PhD}=4$ ), categorized employment situation (employed at $\mathrm{T} 1$ and $\mathrm{T} 2=1$; self-employed at $\mathrm{T} 1$ and $\mathrm{T} 2=2$; unemployed at $\mathrm{T} 1$ and $\mathrm{T} 2=3$; became employed at $\mathrm{T} 2=4$; became (temporarily) unemployed at $\mathrm{T} 2=5$; retired $=6$; on sick leave at $\mathrm{T} 1$ and $\mathrm{T} 2=7$; went on sick leave at $\mathrm{T} 2=8$; student $=9)$, categorized delta coffee usage $(<-3=1 ;-3$ to $<0=$ $2 ; 0=3 ;>0$ to $3=4 ;>3=5$ ), T1 salutogenic effect and T1 COVID-19-infection. To correlate fluctuating quantitative variables such as PSQI total score, vigorous activity score, moderate activity score and sitting time, a delta-score of each of these variables was computed by subtracting the T1-score from the T2-score. To subsequently also gain the possibility to correlate these variables with the possible moderating qualitative variables, these delta-scores were categorized as follows: delta of the PSQI total score $(<0=1 ; 0=2 ;>0=3)$, a delta of the vigorous activity score $(<-31=1 ;-30$ to $30=2$; $>31=3)$, a delta of the moderate activity score $(<-31=1 ;-30$ to $30=2 ;>31=3)$ and delta of the sitting time $(<-31=1 ;-30$ to $30=2 ;>31=3)$. As in the present study, a large number of tests was run, controlling for the alpha inflation was needed. We controlled the proportion of type I errors among all rejected null hypotheses by setting the False Discovery Rate (FDR) to 0.08. The FDR was estimated through the procedure described in Storey and Tibshirani (2003). The bootstrap procedure was used to estimate the p0 parameter (Storey et al., 2004) (for a general view on the bootstrap procedures, see Efron and Tibshirani (1993)). In our results, the 0.05 level of significance corresponds to an FDR of 0.08 .

\section{Results}

\section{Sociodemographic results}

The analytic sample $(n=748$; Belgium $=442$, Spain $=183$, Germany $=50$, Italy $=50$, US $=23)$ was $66 \%$ female (Age $=41 \pm 14$ years). The majority of participants were Europeans $(97 \%)$, of which $94 \%$ at least had a secondary school degree and 39\% had at least a bachelor's degree. $2 \%$ of the participants had positively tested on COVID-19 before completing the online survey the first time, while $45 \%$ where not sure whether they had been infected with COVID-19 or not. This degree of COVID-19-positive participants did not change significantly from T1 to T2. 
Table 1. The effect of prolonged COVID-related restrictions on psychological, exercise and general health variables

Non-parametrically tested variables

Sample Baseline Follow-up

size $(n)$ score (T1) score (T2) $P$-value

\begin{tabular}{lllll}
\hline Sleep & & & & \\
PSQI Comp 1 & 746 & $1.2 \pm 0.8$ & $1.1 \pm 0.8$ & 0.159 \\
PSQI Comp 2 & 747 & $1.3 \pm 1.0$ & $1.2 \pm 1.0$ & $0.010^{*}$ \\
PSQI Comp 3 & 748 & $0.9 \pm 0.9$ & $0.9 \pm 0.9$ & 0.953 \\
PSQI Comp 4 & 738 & $0.7 \pm 1.0$ & $0.6 \pm 0.9$ & $0.013^{*}$ \\
PSQI Comp 5 & 747 & $1.1 \pm 0.5$ & $1.0 \pm 0.5$ & $<0.001^{*}$ \\
PSQI Comp 6 & 747 & $0.3 \pm 0.8$ & $0.3 \pm 0.8$ & 0.914 \\
PSQI Comp 7 & 747 & $0.8 \pm 0.7$ & $0.8 \pm 0.7$ & 0.550 \\
Mood State & & & & \\
POMS - Tension & 695 & $1.3 \pm 1.0$ & $1.4 \pm 1.8$ & 0.582 \\
POMS - Anger & 742 & $1.0 \pm 0.9$ & $1.2 \pm 1.4$ & 0.641 \\
POMS - Fatigue & 742 & $1.4 \pm 1.1$ & $1.5 \pm 1.7$ & 0.860 \\
POMS - Depression & 742 & $1.0 \pm 0.9$ & $1.1 \pm 1.5$ & $0.050^{*}$ \\
POMS - Vigour & 742 & $2.0 \pm 0.8$ & $2.3 \pm 2.0$ & 0.252 \\
POMS - Confusion & 695 & $1.2 \pm 1.0$ & $1.3 \pm 1.4$ & 0.421 \\
Stimulating substances & & & & \\
\#Cigarettes/day & 746 & $0.3 \pm 0.8$ & $0.3 \pm 0.8$ & 0.401 \\
\#Glasses beer/day & 703 & $0.5 \pm 1.2$ & $0.5 \pm 1.2$ & 0.397 \\
\#Glasses wine/day & 695 & $0.7 \pm 1.3$ & $0.7 \pm 1.2$ & 0.747 \\
\#Cups of coffee/day & 704 & $2.4 \pm 2.4$ & $2.1 \pm 2.4$ & $<0.001^{*}$ \\
\#Cans of stimulating caffeinated beverages/day & 747 & $0.5 \pm 1.2$ & $0.5 \pm 1.1$ & 0.705 \\
Salutogenic effect & & & & \\
Salutogenic effect (\%) & 564 & 70 & 66 & 0.296 \\
COVID-related questions & & & & \\
COVID-crisis will be one of long duration? & 746 & $4.2 \pm 0.7$ & $4.2 \pm 0.8$ & 0.358 \\
(5-point Likert-type scale) & & & & \\
Trust in the authorities to handle the crisis? & 746 & $3.1 \pm 1.1$ & $3.0 \pm 1.1$ & $<0.001$ \\
(5-point Likert-type scale) & & & & \\
COVID-19 is a serious and life-threatening & 746 & $4.1 \pm 0.9$ & $4.0 \pm 0.9$ & 0.048 \\
disease (5-point Likert-type scale) & & & & 0.564 \\
\% COVID-19 infected & 410 & 2 & 2 & \\
\hline
\end{tabular}

Data are presented as means $\pm \mathrm{SD}$. *Denotes a significant effect of time.

\section{The effect of prolonged COVID restrictions on human functioning and psychophysiology}

The results of prolonged COVID-related restrictions on psychological, exercise and general health variables are summarized in Tables 1 and 2. In terms of sleep, the PSQI total score was found to decrease in time (T1: $6.2 \pm 3.5 ; \mathrm{T} 2: 5.9 \pm 3.6 ; P=0.003$ ). To further understand the underlying cause of this decrease in the PSQI total score, PSQI subcomponent scores were also analysed, as well as quantitative variables such as hours in bed, sleep latency, sleep per night and sleep efficiency. This analysis demonstrated that PSQI subcomponent-scores 2, 4 and 5, respectively 'sleep latency', 'sleep efficiency' and 'sleep disturbance', improved in time (see Table $1 ; P \leq 0.013$ ). This was further confirmed by the 
Table 2. The effect of prolonged COVID-related restrictions on psychological, exercise and general health variables

Parametrically tested variables

Sample size (n) Baseline score (T1) Follow-up score (T2) $P$-value

\begin{tabular}{lcccc} 
Sleep & & & & \\
$\quad$ PSQI Total score & 737 & $6.2 \pm 3.5$ & $5.9 \pm 3.6$ & $0.003^{*}$ \\
Hours in bed (h) & 737 & $8.5 \pm 1.2$ & $8.4 \pm 1.1$ & $<0.001^{*}$ \\
Sleep latency (min) & 719 & $28 \pm 34$ & $28 \pm 34$ & 0.968 \\
$\quad$ Sleep per night (h) & 740 & $7.2 \pm 1.2$ & $7.1 \pm 1.3$ & 0.790 \\
$\quad$ Sleep efficiency (\%) & 733 & $85 \pm 13$ & $86 \pm 14$ & $0.008^{*}$ \\
Physical activity & & & & \\
$\quad$ Vigorous activity score (min) & 482 & $112 \pm 165$ & $131 \pm 228$ & $0.049^{*}$ \\
$\quad$ Moderate activity score (min) & 482 & $152 \pm 232$ & $191 \pm 304$ & $0.004^{*}$ \\
$\quad$ Walking score & 482 & $292 \pm 448$ & $265 \pm 336$ & 0.186 \\
Sitting time & 482 & $455 \pm 216$ & $437 \pm 202$ & $0.048^{*}$ \\
$\quad$ Overall IPAQ score (min) & 482 & $557 \pm 612$ & $587 \pm 557$ & 0.292 \\
Loneliness & & & & \\
$\quad$ UCLA loneliness scale & 747 & $32 \pm 12$ & $32 \pm 13$ & 0.457 \\
State Anxiety & & & & \\
$\quad$ STAI-score (20-80) & 712 & $43 \pm 14$ & & 0.080 \\
\hline
\end{tabular}

Data are presented as mean $\pm \mathrm{SD}$. *Denotes a significant effect of time.

analysis of the quantitative sleep variables (see Table 2). Thus, hours in bed significantly decreased from T1 to T2 $(P<0.001)$, while sleep efficiency increased from T1 to T2 $(P=0.008)$.

Concerning physical activity, the vigorous and moderate activity score both increased from T1 to T2 (see Table 2; $P \leq 0.049$ ), while sitting time decreased (see Table $2 ; P=0.048$ ). The walking score did not significantly differ from $\mathrm{T} 1$ to $\mathrm{T} 2$.

No differences from T1 to T2 were observed in terms of mood, loneliness and state anxiety (see respectively Tables 1 and 2). Only the depression-subscale of the POMS almost significantly increased from $\mathrm{T} 1(1.0 \pm 0.9)$ to $\mathrm{T} 2(1.1 \pm 1.5 ; P=0.050)$.

The usage of stimulating substances such as cigarettes, beer, wine, coffee and caffeinated energy drinks was also followed up from $\mathrm{T} 1$ to $\mathrm{T} 2$. No differences were observed, except for a significant reduction in the daily coffee intake (T1: $2.4 \pm 2.4$ cups; T2: $2.1 \pm 2.4$ cups; $P<0.001$; see Table 1 ).

COVID-related questions revealed that, within our sample size, a general belief was present that the COVID-crisis would be one of long duration and this belief did not change from T1 to T2 (see Table 1). In contrast, the trust in the authorities to handle the crisis $(P<0.001)$ and the belief whether COVID-19 is a serious and life-threatening disease decreased in time (see Table $1 ; P=0.048$ ). Nonetheless, $70 \%$ of our sample size perceived, in some way, a salutogenic effect of the COVID-related lockdown at $\mathrm{T} 1$. This percentage dropped to $66 \%$ in $\mathrm{T} 2$, but this was non-significant (see Table 1).

\section{Moderators of the effects of prolonged COVID restrictions}

In terms of the possible quantitative moderating variables, multiple significant correlations were observed (see Table 3). The delta PSQI total score was found to be negatively correlated with the T1 PSQI total score $(r=-0.350, P<0.001)$, indicating that individuals with a higher (i.e., worser) PSQI total score at the start of the COVID lockdown were more prone to improve in their PSQI 
Table 3. Pearson correlation coefficients between: (A) PSQI total score, delta vigorous activity IPAQ-score, delta moderate activity IPAQ-score, and delta IPAQ-sitting time, and (B) age, T1 overall PSQI-score, Delta PSQI total score, T1 overall IPAQ-score, delta vigorous activity IPAQ-score, delta moderate activity IPAQ-score, delta IPAQ-sitting time, and T1 STAI-score

\begin{tabular}{|c|c|c|c|c|c|c|c|c|}
\hline & \multicolumn{2}{|c|}{$\begin{array}{c}\text { Delta PSQI total } \\
\text { score }\end{array}$} & \multicolumn{2}{|c|}{$\begin{array}{l}\text { Delta vigorous } \\
\text { activity } \\
\text { IPAQ-score }\end{array}$} & \multicolumn{2}{|c|}{$\begin{array}{l}\text { Delta moderate } \\
\text { activity } \\
\text { IPAQ-score }\end{array}$} & \multicolumn{2}{|c|}{$\begin{array}{c}\text { Delta } \\
\text { IPAQ-sitting } \\
\text { time }\end{array}$} \\
\hline & $R$ & $P$-value & $R$ & $P$-value & $R$ & $P$-value & $R$ & $P$-value \\
\hline Age & 0.047 & 0.198 & 0.007 & 0.880 & -0.072 & 0.115 & 0.029 & 0.523 \\
\hline T1 PSQI total score & -0.350 & $<0.001^{*}$ & -0.029 & 0.520 & 0.008 & 0.868 & -0.058 & 0.207 \\
\hline Delta PSQI total score & I & l & -0.017 & 0.707 & -0.044 & 0.336 & 0.096 & $0.035^{*}$ \\
\hline T1 overall IPAQ-score & 0.079 & 0.085 & -0.149 & $0.001 *$ & -0.198 & $<0.001 *$ & 0.049 & 0.286 \\
\hline $\begin{array}{l}\text { Delta vigorous activity } \\
\text { IPAQ-score }\end{array}$ & -0.017 & 0.707 & / & 1 & 0.038 & 0.401 & -0.110 & $0.015^{*}$ \\
\hline $\begin{array}{l}\text { Delta moderate activity } \\
\text { IPAQ-score }\end{array}$ & -0.044 & 0.336 & 0.038 & 0.401 & l & / & -0.126 & $0.005^{*}$ \\
\hline Delta IPAQ-sitting time & 0.096 & $0.035^{*}$ & -0.110 & $0.015^{*}$ & -0.126 & $0.005^{*}$ & l & / \\
\hline T1 STAI-score & -0.092 & $0.012 *$ & -0.016 & 0.732 & 0.087 & 0.057 & -0.040 & 0.382 \\
\hline
\end{tabular}

*Denotes a significant correlation.

total score (i.e., a lower delta PSQI score). The STAI-score at T1 was also observed to be negatively correlated with the delta PSQI total score $(r=-0.092, P=0.012)$. In addition, the delta PSQI total score positively correlated with the delta IPAQ-sitting time $(r=0.096, P=0.035)$, showing that individuals who had a lower PSQI total score at T2 compared to T1 also had a lower amount of time sitting at $\mathrm{T} 2$ compared to T1. In addition, a negative correlation between delta scores of both vigorous and moderate activity with respect to sitting time was found $(r=-0.110, P=0.015$ and $r=-0.126, P=0.005$, respectively, see Table 3). Furthermore, the delta in both vigorous and moderate activity was negatively associated with the overall IPAQ-score at T1 $(r=-0.149, P=0.001$ and $r=-0.198, P<0.001$, respectively, see Table 3), which demonstrates that mostly individuals with a lower overall IPAQ-score in the beginning of the lockdown invested more time in doing vigorous and moderate activity at $\mathrm{T} 2$ than at $\mathrm{T} 1$ of the lockdown.

Finally, in terms of the possible qualitative moderating variables multiple significant Pearson $\chi^{2}$ values were observed as well (see Table 4). Of these, the most interesting results were the significant link between the categorized delta PSQI total scores and the categorized coffee usage $(P=0.030)$, the significant link between the categorized delta vigorous activity and the categorized age $(P=0.004)$ and the significant link between the categorized delta sitting time and gender $(P=0.049)$. Interpreting these significant links revealed that $71 \%$ did not change their coffee drinking habits from $\mathrm{T} 1$ to $\mathrm{T} 2$, while $16 \%$ decreased their daily coffee intake by $1-3$ cups and $9 \%$ increased their daily coffee intake by 1-3 cups. Interestingly, of the $16 \%$ that decreased their daily coffee intake, $55 \%$ improved (i.e., decreased) their PSQI total score. In contrast, of the $9 \%$ that increased their daily coffee intake, only $28 \%$ improved (i.e., decreased) their PSQI total score. Regarding the link between vigorous activity and age, $24 \%$ of the participants increased the time invested in vigorous activity during a week. Within this $24 \%$, the age group most represented (49\%) is between 25 and 44 years old, (other groups: $<25=11 \% ; 45-64=32 \% ;>64=8 \%$ ). In terms of the link between sitting time and gender, $44 \%$ of the women reduced their sitting time, while only $34 \%$ of the men did the same. 
Table 4. Chi-square distributions between: (A) categorized delta PSQI total score, categorized delta vigorous activity IPAQ-score, categorized delta moderate activity IPAQ-score, and categorized delta IPAQ-sitting time, and (B) country, categorized age, gender, categorized educational level, categorized employment situation, categorized coffee usage, T1 salutogenic effect, and T1 COVID-19-infection

\begin{tabular}{|c|c|c|c|c|c|c|c|c|}
\hline & \multicolumn{2}{|c|}{$\begin{array}{l}\text { Categorized } \\
\text { delta PSQI } \\
\text { total score }\end{array}$} & \multicolumn{2}{|c|}{$\begin{array}{c}\text { Categorized } \\
\text { delta vigorous } \\
\text { activity } \\
\text { IPAQ-score }\end{array}$} & \multicolumn{2}{|c|}{$\begin{array}{c}\text { Categorized } \\
\text { delta moderate } \\
\text { activity } \\
\text { IPAQ-score }\end{array}$} & \multicolumn{2}{|c|}{$\begin{array}{c}\text { Categorized } \\
\text { delta } \\
\text { IPAQ-sitting } \\
\text { time }\end{array}$} \\
\hline & Value & $P$-value & Value & $P$-value & Value & $P$-value & Value & $P$-value \\
\hline Country & 9.9 & 0.275 & 9.9 & 0.274 & 20.3 & $0.009^{*}$ & 24.1 & $0.002 *$ \\
\hline Categorized age & 4.6 & 0.599 & 19.4 & $0.004 *$ & 1.9 & 0.926 & 4.4 & 0.619 \\
\hline Gender & 0.5 & 0.796 & 0.5 & 0.771 & 0.9 & 0.628 & 6.0 & $0.049 *$ \\
\hline Categorized educational level & 9.1 & 0.336 & 7.8 & 0.450 & 2.7 & 0.953 & 4.4 & 0.816 \\
\hline Categorized employment situation & 16.5 & 0.417 & 10.0 & 0.866 & 8.6 & 0.928 & 20.4 & 0.203 \\
\hline Categorized coffee usage & 17.0 & $0.030 *$ & 8.0 & 0.430 & 7.8 & 0.451 & 4.5 & 0.805 \\
\hline T1 salutogenic effect & 0.5 & 0.772 & 2.7 & 0.263 & 5.0 & 0.083 & 1.9 & 0.390 \\
\hline T1 COVID-19-infection & 3.4 & 0.179 & 0.5 & 0.777 & 1.9 & 0.380 & 3.9 & 0.141 \\
\hline
\end{tabular}

*Denotes a significant Pearson $\chi^{2}$.

\section{Discussion}

The most important findings of the present study are as follows: (1) A prolonged COVID-related lockdown resulted in improved subjective sleep quality, i.e., a decreased PSQI total score, related to an increase in subjective sleep efficiency and a decrease in sleep latency and disturbance; (2) A prolonged COVID-related lockdown resulted in a decrease in weekly sitting time and an increase in the weekly amount of moderate and vigorous physical activity (as measured with the IPAQ); (3) In terms of intake of stimulating substances, only the daily coffee intake decreased from T1 to T2; (4) No major differences in mood, anxiety and loneliness related to a prolonged COVID-related lockdown were observed over the time period analysed; (5) $70 \%$ of our sample size perceived, in some way, a salutogenic effect of the COVID-related lockdown; (6) A lower amount of sitting time seems to be linked to the observed improvements in sleep and increased weekly vigorous and moderate physical activity. Moreover, a greater relative number of women compared to men decreased their amount of weekly sitting time.

\section{The effect of prolonged COVID restrictions on human functioning and psychophysiology}

In the present study, it was observed that a prolonged COVID-related lockdown resulted in multiple positive effects in psychological, exercise and general health variables with no apparent negative effects. As such, we cannot confirm the hypothesized negative effect of a prolonged COVID-related lockdown on sleep- and exercise-related variables.

In terms of sleep, we expected a decrease in sleep quality with prolonged confinement. Pépin et al. (2021) reported that the COVID-19 lockdown, and the associated dramatic societal change, delayed bedtime and wake-up time schedules and also attenuated the differences in the timing of sleep between weekdays and weekends. These changes in sleep patterns appeared rapidly after the start of the COVID-19 lockdown (Pépin et al., 2021). Moreover, Pépin et al. (2021) also observed a COVID-19 lockdown-related prolonged sleep latency, a decreased slow-wave sleep and increased light and rapid eye movement sleep. The rapid change in sleep patterns observed by Pépin et al. (2021), was further confirmed by the data of Cellini et al. (2020). Cellini et al. (2020) reported that 
by the second week of the lockdown, people (i.e., both students and workers) were going to bed ( $\sim 41$ min) and waking up $(\sim 1 \mathrm{~h})$ later, and they were also spending more time in bed (workers: $\sim 26 \mathrm{~min}$, students: $\sim 5 \mathrm{~min}$ ). Paradoxically, however, Cellini et al. (2020) also found a significant COVID lockdown-related increase in the PSQI score (i.e., a lower sleep quality). Cellini et al. (2020) put forward: reduced exposure to sunlight, reduced physical activity, psychological distress, lack of social zeitgebers (e.g., regular work schedules and social activities) and changes in living conditions as important factors that might explain the negative effect of the COVID-19 lockdown on sleep habits and quality. In addition, research from Beck et al. (2021) also presented questionnaire data that suggested an association between the COVID-19 lockdown and severe sleep disorders. Based on these data there seemed to be a pressing need to develop strategies to reduce the adverse psychological impacts of the COVID-19 lockdown. Subsequently, this need was also stressed in the study of Cellini et al. (2020). Although the present study cannot determine whether the ad hoc-developed strategies to reduce adverse psychological impacts of the COVID-19 lockdown were in play, we did observe that a few weeks after the start of the lockdown the overall PSQI score improved compared to during the start of the lockdown. The fact that this improvement in the overall PSQI score was related to a decreased sleep latency and an increased sleep efficiency (i.e., the sleep variables that deteriorated in the first week of the lockdown) might suggest that the improved sleep was possibly related to a renormalization of the sleep schedules. Thus, our results suggest that after the first (negative) impact of the COVID-related lockdown on sleep schedules, people were generally able to partly bounce back and improve the organization of their sleep schedules.

For physical exercise, we expected a decrease in the amount of physical activity. Belavý et al. (2013) followed up the physical activity of six individuals in the Mars500 study (i.e., 520 days of isolation) and observed that physical activity progressively decreased over the course of the isolation period. Even after 40 days of isolation (i.e., a comparable time frame as the time frame between the start of the lockdown and T2 in the present study), the 'acceleration variance' was already significantly decreased (i.e., subjects changed body position less and/or changed their speed of walking less) (Belavý et al., 2013). In the present study, we expected a similar change in 'lifestyle' with prolonged lockdown measures, mainly due to the closure of gyms and sport clubs and the travel restrictions. However, despite the COVID-related lockdown measures, our participants reported an increase in physical activity. We speculate that, even though the variety of sports that could be practised had been greatly reduced, the lack of alternative types of activities to engage in (i.e., social activities were also greatly restricted) resulted in an increased opportunity to engage in physical activity. Physical activities such as running and cycling have greatly increased during the COVID-19 lockdown (Lock, 2020), and unlike in the Mars500 study, most of the participants in the present study were still able to go out of their houses and find a way to exercise recreationally.

\section{Moderators of the effects of prolonged COVID restrictions}

To assess which moderators could have possibly played a role in the positive impact of a prolonged COVID-related lockdown on physical activity and sleep, correlational statistics were performed with both quantitative and qualitative variables.

Of the many possible moderators tested, those significant appeared to be (1) the reduced amount of sitting time; (2) the daily coffee usage; (3) age; and (4) gender. Of these four moderators, only sitting time was found to play a role in both improved sleep and improved physical activity. A logical negative correlation between sitting time and vigorous as well as moderate physical activity was found. In addition, decreased sitting time from T1 to T2 was linked with improved (i.e., lower) overall PSQI scores. This finding immediately emphasizes the already-established link between physical activity and sleep quality (Schneider et al., 2010). Within the confinement literature, Schneider et al. (2010) and Abeln et al. (2015) demonstrated that exercise is a suitable method to counteract psycho-physiological deconditioning during confinement. The present study further supports this conclusion. In addition to the reduction in the amount of sitting time, a decrease in daily coffee usage was also related to an improved 
total PSQI score. This link has been previously established (Clark and Landolt, 2017; Weibel et al., 2021). Caffeine is known to be an adenosine receptor antagonist, which blocks $A_{1}$ and $A_{2 A}$ adenosine receptors in the central nervous system, and as such can attenuate the increase in sleep pressure during wakefulness and negatively impact sleep quality (Clark and Landolt, 2017). Daily coffee intake is known to be related to the workplace, since coffee sociability at work is typically an integral part of workplace culture (Stroebaek, 2013). Subsequently, the COVID-19 lockdown disrupting the normal work and workplace routines, could explain the observed decrease in daily coffee intake in the present study.

In terms of age, individuals aged between 25 and 44 years old were the most represented in the $24 \%$ that increased their weekly vigorous activity from T1 to T2. This finding appears to be logical, as this age group, in normal circumstances, is expected to be heavily involved in multiple work-, social- and parental-related activities, not leaving enough time for other activities such as physical activities. With the COVID-19 related restrictions resulting in the temporary removal of those obligations, it seems logical that this age group benefited the most in terms of being able to spend the newly available free time in performing physical activity. Concerning the role of gender, from our subject population, $44 \%$ of the women reduced their sitting time, while only $34 \%$ of the men did the same. This indicates that women experienced more positive effects of the lockdown in terms of physical activity. These results support previous findings in an Antarctic overwintering study from Steinach et al., 2016. Steinach et al. (2016) evaluated the physical activity levels throughout seven Antarctic overwintering campaigns and observed that physical activity significantly dropped for men but not for women. Nevertheless, Steinach et al. (2016) concluded that women had a higher susceptibility to psychosocial stress and changes in environmental circadian rhythm during long-term isolation in Antarctica. These conclusions were derived from the finding that the sleep quality of women during Antarctic overwintering decreased to a greater extent than of men (Steinach et al., 2016).

\section{Limitations and future directions}

The foremost limitation was certainly the different sample sizes between countries. Despite similar recruiting procedures in every country, some countries had a considerably larger sample size than others. This of course restricted the possibilities in comparing the effects of the COVID lockdown on psychological, exercise and general health variables between countries. Subsequently, it was decided to interpret the COVID lockdown effects in general on this worldwide, European-dominated sample and not attempt to elucidate the subtle differences that the country-specific COVID-restrictions could have provided. In the end, the authorship team believed that the COVID-related restrictions in each country resembled each other sufficiently to have a similar impact on social-, work- and lifestyle-related habits. Future research on COVID lockdown-related data might however greatly benefit from large sample size studies with a balanced cohort between countries to evaluate whether specific differences in COVID-related restrictions triggered a different impact of the COVID lockdown on human functioning. The present results only picture changes observed within the first weeks of the first lockdown and encourage future research to explore the possible moderating role of daily coffee usage, physical activity and gender in the effect of prolonged isolation on sleep and physical activity. Schneider et al. (2010) already demonstrated that brain activity changes might mediate the positive effects of physical activity on psycho-physiological functioning during prolonged isolation (i.e., prolonged isolation seems to be related to a depression of brain cortical activity, and exercise seems to activate brain cortical processes). Moreover, they also emphasized that exercise intensity and duration, as well as exercise preferences need to be taken into account to optimally affect brain cortical activity and as such human psycho-physiological functioning (Schneider et al., 2010). Undoubtedly COVID-lockdown-related studies that specifically focus on the impact of the lockdown measures (i.e., the situation in which most analogies with space missions are present) on human psycho-physiological functioning will be of great value for space research. 


\section{Conclusion}

Compared to 3 weeks into the first COVID-imposed lockdown, 6-weeks after the start of the first COVID-imposed lockdown, physical activity and subjective sleep scores were positively impacted. This positive impact was associated with a decrease in sitting time and, specifically for sleep, with an increase in vigorous and moderate physical activity and a decrease in daily coffee intake. Individuals aged between 25 and 44 years old were the most represented in the $24 \%$ that increased their weekly vigorous activity from T1 to T2. A possible role of gender in the decrease in sitting time throughout a prolonged lockdown was also observed and substantiates previous results demonstrating that women might alter their physical activity during prolonged isolation differently from men. The present, large sample size study confirms exercise is a promising method to counteract psycho-physiological deconditioning during prolonged isolation which needs to be considered for future space missions but also pandemic scenarios on Earth.

Acknowledgements. The authors declare that the results of the study are presented clearly, honestly and without fabrication, falsification, or inappropriate data manipulation. They thank the participants for their engagement in this study. They also thank colleagues Stefano Sdoia and Pierpaolo Zivi for their help with the data analysis.

Conflicts of interest. None.

\section{References}

Abeln V, MacDonald-Nethercott E, Piacentini MF, Meeusen R, Kleinert J, Strueder HK and Schneider S (2015) Exercise in isolation--a countermeasure for electrocortical, mental and cognitive impairments. PLoS One 10, e0126356.

Bartone PT, Krueger GP and Bartone JV (2018) Individual differences in adaptability to Isolated, confined, and extreme environments. Aerospace Medicine and Human Performance 89, 536-546.

Basner M, Dinges DF, Mollicone DJ, Savelev I, Ecker AJ, Di Antonio A, Jones CW, Hyder EC, Kan K, Morukov BV and Sutton JP (2014) Psychological and behavioral changes during confinement in a 520-day simulated interplanetary mission to Mars. PLoS One 9, e93298.

Beck SL, Schwartz AL, Towsley G, Dudley W and Barsevick A (2004) Psychometric evaluation of the Pittsburgh sleep quality index in cancer patients. Journal of Pain and Symptom Management 27, 140-148.

Beck F, Léger D, Fressard L, Peretti-Watel P, Verger P and Group C (2021) Covid-19 health crisis and lockdown associated with high level of sleep complaints and hypnotic uptake at the population level. Journal of Sleep Research 30, e13119.

Belavý DL, Gast U, Daumer M, Fomina E, Rawer R, Schieß1 H, Schneider S, Schubert H, Soaz C and Felsenberg D (2013) Progressive adaptation in physical activity and neuromuscular performance during 520d confinement. PLoS One 8, e60090.

Buysse DJ, Reynolds CF, Monk TH, Berman SR and Kupfer DJ (1989) The Pittsburgh sleep quality Index: a new instrument for psychiatric practice and research. Psychiatry Research 28, 193-213.

Cellini N, Canale N, Mioni G and Costa S (2020) Changes in sleep pattern, sense of time and digital media use during COVID-19 lockdown in Italy. Journal of Sleep Research 29, e13074.

Clark I and Landolt HP (2017) Coffee, caffeine, and sleep: a systematic review of epidemiological studies and randomized controlled trials. Sleep Medicine Reviews 31, 70-78.

Craig CL, Marshall AL, Sjöström M, Bauman AE, Booth ML, Ainsworth BE, Pratt M, Ekelund U, Yngve A, Sallis JF and Oja P (2003) International physical activity questionnaire: 12-country reliability and validity. Medicine \& Science in Sports \& Exercise 35, 1381-1395.

Efron B and Tibshirani R (1993) An introduction to the Bootstrap. New York: Chapman \& Hall.

Fillion L and Gagnon P (1999) French Adaptation of the shortened version of the profile of mood states. Psychological Reports 84, 188-190.

Gemignani A, Piarulli A, Menicucci D, Laurino M, Rota G, Mastorci F, Gushin V, Shevchenko O, Garbella E, Pingitore A, Sebastiani L, Bergamasco M, L'Abbate A, Allegrini P and Bedini R (2014) How stressful are 105 days of isolation? Sleep EEG patterns and tonic cortisol in healthy volunteers simulating manned flight to Mars. International Journal of Psychophysiology 93, 211-219.

Julian LJ (2011) Measures of anxiety: state-trait anxiety inventory (STAI), Beck anxiety inventory (BAI), and hospital anxiety and depression scale-anxiety (HADS-A). Arthritis Care Res (Hoboken) 63(suppl. 11), S467-S472.

Kanas N (1998) Psychosocial issues affecting crews during long-duration international space missions. Acta Astronautica 42, 339-361.

Keller N, McHenry N, Duncan C, Johnston A, Whittle R, Koock E, Bhattacharya S, De la Torre G, Ploutz-Snyder L, Sheffield-Moore M, Chamitoff G and Diaz-Artiles A (2021) Augmenting Exercise Protocols with Interactive Virtual Reality Environments. IEEE Aerospace Conference. 
Lock O (2020) Cycling behaviour changes as a result of COVID-19: a survey of users in Sydney, Australia. Transport Findings.

Mairesse O, MacDonald-Nethercott E, Neu D, Tellez HF, Dessy E, Neyt X, Meeusen R and Pattyn N (2019) Preparing for Mars: human sleep and performance during a 13 month stay in Antarctica. Sleep 42(1).

Matsangas P, Shattuck NL, Heinicke C and Dunn J (2017) Sleep Patterns of Crewmembers in Mission IV of the Hawaii Space Exploration Analog and Simulation (HI-SEAS): A Pilot Study. Human Factors and Ergonomics Society 2017 Annual Meeting.

McNair DM, Lorr M and Droppleman LF (1971) Manual for the profile of mood states (POMS). San Diego: Educational and Industrial Testing Service.

Mimoso D, Gil Calle E, Martin Estrana V, Lizy-Destrez S and Roy R (2020) Towards Teleoperation Performance and Psychophysiological State Assessment in the SIRIUS-19 Analog Campaign. IAC - The CyberSpace Edition, Paris, France.

Pagel JI and Choukèr A (2016) Effects of isolation and confinement on humans-implications for manned space explorations. The Journal of Applied Physiology 120, 1449-1457.

Pattyn N, Mairesse O, Cortoos A, Marcoen N, Neyt X and Meeusen R (2017) Sleep during an Antarctic summer expedition: new light on "polar insomnia". The Journal of Applied Physiology 122, 788-794.

Pattyn N, Van Puyvelde M, Fernandez-Tellez H, Roelands B and Mairesse O (2018) From the midnight sun to the longest night: sleep in Antarctica. Sleep Medicine Reviews 37, 159-172.

Pépin JL, Bailly S, Mordret E, Gaucher J, Tamisier R, Ben Messaoud R, Arnal PJ and Mignot E (2021) Greatest changes in objective sleep architecture during COVID-19 lockdown in night-owls with increased REM sleep. Sleep 44(9).

Russell D, Peplau LA and Ferguson ML (1978) Developing a measure of loneliness. Journal of Personality Assessment 42, $290-294$.

Sandal GM, van de Vijver FJR and Smith N (2018) Psychological hibernation in Antarctica. Frontiers in Psychology 9, $22-35$.

Schneider S, Brümmer V, Carnahan H, Kleinert J, Piacentini MF, Meeusen R and Strüder HK (2010) Exercise as a countermeasure to psycho-physiological deconditioning during long-term confinement. Behavioural Brain Research 211, $208-214$.

Scott JPR, Weber T and Green DA (2019) Introduction to the frontiers research topic: optimization of exercise countermeasures for human space flight - lessons from terrestrial physiology and operational considerations. Frontiers in Physiology $10,173$.

Spielberger C (1983) Manual for the State-Trait Inventory STAI (Form Y). Palo Alto. Consulting Psychologists Press.

Steinach M, Kohlberg E, Maggioni MA, Mendt S, Opatz O, Stahn A and Gunga HC (2016) Sleep quality changes during overwintering at the German Antarctic Stations Neumayer II and III: the gender factor. PLoS One 11, e0150099.

Storey JD and Tibshirani R (2003) Statistical significance for genomewide studies. Proceedings of the National Academy of Sciences of the United States of America 100, 9440-9445.

Storey J, Taylor J and Siegmund D (2004) Strong control, conservative point estimation and simultaneous conservative consistency of false discovery rates: a unified approach. Journal of the Royal Statistical Society B 66, 187-205.

Stroebaek PS (2013) Let's have a cup of coffee! coffee and coping communities at work. Symbolic interaction 36, $381-397$.

Terry PC, Lane AM and Fogarty GJ (2003) Construct validity of the profile of mood states-adolescents for use with adults. Psychology of Sport and Exercise 4, 125-139.

Urbina D and Charles R (2014) Symposium keynote: enduring the isolation of interplanetary travel. A personal account of the Mars500 mission. Acta Astronautica 93, 374-383.

Wald F and Mellenbergh G (1990) De verkorte versie van de Nederlandse vertaling van de profile of mood states (POMS). Nederlands Tijdschrift Voor De Psychologie En Haar Grensgebieden 45(2), 86-90.

Weibel J, Lin YS, Landolt HP, Kistler J, Rehm S, Rentsch KM, Slawik H, Borgwardt S, Cajochen C and Reichert CF (2021) The impact of daily caffeine intake on nighttime sleep in young adult men. Scientific Reports 11, 4668.

Zivi P, De Gennaro L and Ferlazzo F (2020) Sleep in isolated, confined, and extreme (ICE): a review on the different factors affecting human sleep in ICE. Frontiers in Neuroscience 14, 851. 